\title{
Michel Eude, un acteur « discret » de la Société des études robespierristes
}

Michel Eude, a « discreetmember » of the Société des études robespierristes

\section{Clyde Plumauzille}

\section{(2) OpenEdition \\ 1 Journals}

\section{Édition électronique}

URL : https://journals.openedition.org/ahrf/11090

DOI : 10.4000/ahrf.11090

ISSN : 1952-403X

Éditeur :

Armand Colin, Société des études robespierristes

\section{Édition imprimée}

Date de publication : 1 septembre 2008

Pagination : 129-143

ISBN : 978-2-200-92515-4

ISSN : 0003-4436

Référence électronique

Clyde Plumauzille, "Michel Eude, un acteur « discret » de la Société des études robespierristes », Annales historiques de la Révolution française [En ligne], 353 I juillet-septembre 2008, mis en ligne le 01 septembre 2011, consulté le 01 juillet 2021. URL : http://journals.openedition.org/ahrf/11090 ; DOI : https://doi.org/10.4000/ahrf.11090

Ce document a été généré automatiquement le 1 juillet 2021.

Tous droits réservés 


\title{
Michel Eude, un acteur « discret » de la Société des études robespierristes
}

\author{
Michel Eude, a « discreetmember » of the Société des études robespierristes
}

\author{
Clyde Plumauzille
}

1 Il reste peu de traces aujourd'hui du parcours de Michel Eude dans les arcanes de l'histoire révolutionnaire du vingtième siècle. Ce dernier a pourtant traversé les grands bouleversements historiographiques de notre passé proche, tout en y participant indirectement par le biais de son activité au sein de la Société des études robespierristes et de son organe éditorial, les Annales historiques de la Révolution française.

2 Ce centenaire nous fournit d'ailleurs l'occasion de revenir sur l'importance de cette plateforme intellectuelle qui porte encore aujourd'hui tant d'historiens et tant d'idées. Les AHRF, lieu majeur pour un historien de faible visibilité comme le fut Michel Eude, conservent la trace indélébile de ses publications, permettant de poser des théories sur ce nom oublié. Elles furent aussi le lieu d'une connaissance d'homme à homme, où le nom de Michel Eude perdure comme souvenir - il est vrai souvent lointain - parmi certains de ses membres. Aussi la Société des études robespierristes est autant aux yeux des générations actuelles un lieu d'histoire, qu'un lieu qui a fait l'histoire, car elle nous permet de nous confronter aux multitudes humaines qui ont bâti notre historiographie.

3 J'ai découvert Michel Eude dans les cartons d'archives de l'Institut d'histoire de la Révolution française, un premier contact très concret du produit papier de ses pensées. Des dossiers poussiéreux émergeaient cependant distinctement un ensemble de petits fascicules identiques estampillés du bonnet phrygien surmontant les faisceaux républicains : les exemplaires d'auteur des premiers articles publiés par Michel Eude au sein des Annales historiques de la Révolution française sur Robespierre et la commune robespierriste. C'est alors que le nom se fit historien, un historien repérable grâce à ce passage dans cette revue. C'est dans le sillage de celle-ci, portant l'essentiel des publications de Michel Eude et surtout l'essentiel de sa visibilité d'historien, que s'est 
construit mon travail d'archivage au sein de l'IHRF ${ }^{1}$. Une visibilité qui reste néanmoins des plus floues dans la " galerie des ancêtres » de la Société des études robespierristes.

Dernier élément introductif sur ce personnage, il faut également ajouter à ce déficit de visibilité, une position marginale dans le champ idéologique de la SER dans les années 1950, 1960, 1970. D'obédience plutôt conservatrice, voire carrément réactionnaire quand il s'exprimait en privé avec ses collègues de la Faculté de Rouen, Michel Eude dérogea en effet fortement aux théories et aux pratiques de l'école révolutionnaire marxiste. Cependant, ses inclinations politiques n'apparaissent pas dans le corps même de son œuvre d'historien, car ce dernier ne s'est jamais engagé dans les grands combats historiographiques de son époque. Néanmoins, sa présence au sein de la SER témoigne de la pluralité intellectuelle qui coexistait au sein de la société. Cette dernière a permis autant de fédérer les études révolutionnaires que de les confronter en vue de susciter le débat. Mais la SER a également donné implicitement la possibilité à ses membres de ne pas participer à ces débats, pourtant perçus comme une sorte de marque de fabrique politique et identitaire d'une société de réflexion révolutionnaire.

5 Michel Eude, personnage émanant au détour des papiers de l'IHRF et des AHRF, est ainsi le prétexte fondateur de cet article, le fil rouge que nous suivrons afin de participer au détail de la richesse de la SER et des hommes qui s'y sont associés. Il s'agit donc de jeter ici quelques pistes de réflexion sur une trajectoire individuelle et un cheminement d'historien en constante relation avec le monde des études robespierristes. Relation qui permet de mettre en valeur l'importance du rôle joué par la société pour la promotion intellectuelle, voire professionnelle, de ses membres, mais aussi de souligner un représentant de cette masse d'adhérents plus ou moins anonymes qui ont accompagné la société.

6 L'itinéraire intellectuel et professionnel de Michel Eude rejoint les trajectoires classiques de bon nombre des historiens et acteurs de la SER. Il convient aussi d'ajouter en arrière-plan de cet itinéraire, certains éléments biographiques qui participent à la formation historique de Michel Eude et permettent ainsi de saisir le contexte de cette "génération des extrêmes" qui connut les guerres mondiales et l'avènement des grandes idéologies.

7 Deux composantes essentielles ont par ailleurs largement contribué à modeler l'univers intellectuel de Michel Eude: tout d'abord une formation universitaire où dans un premier temps l'élève Michel Eude étudia sous la direction des grandes figures du renouveau des études révolutionnaires comme Albert Mathiez et Georges Lefebvre, mais aussi un itinéraire familial et personnel qui inscrit ses positions historiographiques, dans une tradition plutôt militaire et conservatrice.

8 À titre préalable il convient d'évoquer rapidement les difficultés de documentation auxquelles nous nous trouvons confrontés dès qu'il s'agit de retracer la biographie d'un personnage on ne peut plus discret dans les archives. De plus, les dossiers d'informations le concernant ne sont pas encore accessibles au public que ce soit au Ministère de l'Éducation nationale ou au CNRS. De ce fait, notre point de départ sera la notice nécrologique de Michel Eude rédigée par Jean-René Suratteau dans les AHRF ${ }^{2}$. Suratteau nous offre la vision d'un membre de la SER ayant connu Michel Eude - avec une certaine sympathie peut-être - et ayant pu également se renseigner auprès de la famille de ce dernier. On voit donc ici un exemple significatif de l'éclairage procuré par la SER et les AHRF sur le travail et la personne de Michel Eude dans le monde de la recherche. Ce dernier a effectué un parcours universitaire plutôt semblable à de 
nombreux membres de la SER. Intégrant l'École normale supérieure de la rue d'Ulm en 1929, il y fait la rencontre décisive d'Albert Mathiez dont il fut, selon Jean-René Suratteau, l'un des élèves favoris, pour en sortir agrégé d'histoire et de géographie en 1933. Après son service militaire, devenu pensionnaire de la Fondation privée Thiers de 1934 à 1936, tout comme l'avait été avant lui Albert Mathiez, il s'engage dans une histoire politique de la Convention montagnarde. Michel Eude s'inscrit donc par sa recherche dans les thèmes de prédilection de la SER. C'est probablement Albert Mathiez, président-fondateur de la Société des études robespierristes, qui lui fit découvrir l'univers social, intellectuel et éditorial des AHRF, permettant par ce «brillant patronage " la publication de son premier article de fond tiré de son mémoire ${ }^{3}$ présenté à la Faculté des Lettres de Paris en 1932 et intitulé Études sur la Commune robespierriste. Cet article paraîtra en plusieurs séquences, entre 1932 - date à laquelle il adhère à la SER - et 1936. On a ici un bon exemple de l'importance du rôle des membres directeurs de la SER qui servent en quelque sorte d'» agents de recrutement », et de celui joué par les "maîtres » et directeurs de recherche auprès de leurs étudiants, intermédiaires essentiels entre le monde universitaire et le monde des sociétés de pensée. Suite au décès d'Albert Mathiez en 1932, la notice de Suratteau se fait plus discrète concernant la relation de Michel Eude avec son "nouveau » maître Georges Lefebvre, cependant celui-ci continua de publier régulièrement dans les AHRF de 1932 à 1937 , essentiellement dans la rubrique "Glanes » sous forme de diverses brèves. De plus, Georges Lefebvre prenant aussi la suite d'Albert Mathiez à la présidence de la SER, remplit à son tour une position permettant à Michel Eude d'occuper une place de choix dans le vivier des jeunes historiens de son époque: ce dernier entre d'ailleurs brièvement au comité directeur de la SER en 1940. Cependant, la relation entre Michel Eude et son directeur Georges Lefebvre semble avoir été plutôt distante, le silence de la notice nécrologique est corroboré par le silence matériel du fonds Eude qui ne contient aucune trace du lien universitaire et sociétaire qui a pourtant uni les deux hommes. Il faut garder ici à l'esprit la position conservatrice de Michel Eude, celle-ci ayant pu être un élément d'une certaine "mésentente cordiale» intellectuelle avec ce maître de sensibilité marxiste, et ce d'autant plus que le sujet de travail choisi était le Comité de Sûreté générale, organe politique et policier du gouvernement montagnard, objet pouvant susciter des débats idéologiques tumultueux. Cette relation explique peut-être pourquoi Michel Eude, après avoir débuté en tant que jeune chercheur sa collaboration aux AHRF par un article de réflexion sur Robespierre -personnage emblématique de la revue - très certainement impulsé par le patronage bienveillant d'Albert Mathiez, se soit limité par la suite à la publication de notices annexes dans les rubriques «Glanes » au sein de la revue les années suivantes.

9 Michel Eude a donc reçu une formation universitaire sous la tutelle des grands historiens de la Révolution de son temps, mais aussi sous la tutelle de membres actifs, voire fondateurs dans le cas d'Albert Mathiez, du mouvement de la SER. Les AHRF ont accompagné tous les pas de Michel Eude dans l'écriture de l'histoire et ce d'autant plus que l'on sait que ce dernier n'achèvera jamais sa thèse d'État. L'apport scientifique de sa formation se traduit essentiellement dans une méthode rigoureuse observée à l'égard de ses sources. Le dépouillement de son corpus archi-vistique sur le Comité de Sûreté générale est on ne peut plus minutieux, reprenant en note l'intégralité du catalogue du fonds des suspects, il le soumit ensuite à plusieurs classements thématiques - le tout bien évidemment noté à la main inlassablement le long de plusieurs milliers de feuillets. C'est ce corpus documentaire qui est d'ailleurs conservé à 
l'IHRF, constituant un précieux outil de consultation du fonds des suspects et des arrêtés du Comité de Sûreté générale établi par Pierre Caron, ainsi qu'une première approche par les sources du Comité de Sûreté générale.

Ce legs méthodologique et cette tutelle prestigieuse sont peut-être le principal dénominateur commun qui unit Michel Eude au monde de la SER. Les «pères fondateurs » des études robespierristes que furent Albert Mathiez et Georges Lefebvre furent tout à la fois un moteur pour le travail de ce dernier ainsi qu'un héritage à partager avec l'ensemble de la société.

Penchons-nous à présent sur le cadre familial de Michel Eude, la notice de Jean-René Suratteau faisant office de point de départ biographique. Michel Eude est né à Paris dans une famille d'officier et d'origine normande. Cet arrière-plan familial et militaire est à mettre en relation avec l'expérience que fera Michel Eude - comme nombre de ses contemporains de la SER, notamment Albert Soboul ou encore Jean-René Suratteau - de la Seconde Guerre mondiale. On parle parfois d'une "génération 1905 » qui a subi les conséquences de la Première Guerre mondiale et participé directement à la seconde : l'expérience de la guerre est à prendre en compte dans toute analyse de ces historiens qui ont fait les Annales dans le courant des années 1950-1960. Mobilisé à l'âge de 30 ans, il est fait prisonnier en juin 1940 puis libéré, après quatorze mois de captivité, pour raison de santé. Il reprend alors un emploi de professeur dans la zone française occupée : poste de fonctionnaire de l'État vichyssois et poste d'enseignant d'histoire au cœur de la tourmente de la Révolution nationale. On ne sait rien de cette période. Dans ses archives personnelles, une parenthèse entoure la Seconde Guerre mondiale. Peutêtre en saurons-nous un peu plus dès la mise à disposition de son dossier d'enseignant en 2019. On ne peut s'empêcher néanmoins de comparer comme un "clair-obscur " l'itinéraire de ce dernier avec celui d'un Albert Soboul révoqué de l'Éducation nationale en 1942, et qui dès le printemps 1941 est l'un des organisateurs du Front national des étudiants du Midi participant activement à la résistance intérieure.

$\mathrm{Si}$, par son parcours universitaire, on comprend que Michel Eude se tourne vers l'enseignement, par son contexte familial militaire, on comprendra également qu'il soit, dès 1945 détaché, sur sa propre motivation, au Gouvernement militaire de la zone française d'occupation à Baden-Oos. Il restera alors sept années en Allemagne, employé à la division de l'Information qui coordonne, encadre et diffuse les nouvelles en provenance de l'Allemagne française vers le territoire français. Dans le cadre de cette occupation professionnelle, il s'investit intellectuellement dans les publications de la zone française d'occupation, et dirige la revue Réalités allemandes ${ }^{5}$ publiée par le gouvernement militaire de la zone d'occupation qui se veut autant "une revue d'information, qu'un instrument de travail pour tous les Français de la zone et les Français de la France ». Une revue franco-française donc - qui laisse peut-être paraître une pointe de chauvinisme pour faire face au "syndrome de la honte» de la collaboration vichyssoise - et une revue développée et publiée par des membres du gouvernement militaire. La direction de cette revue par Michel Eude, cas unique dans son parcours professionnel, s'effectue dans le cadre militaire, lui laissant une marge de manœuvre intellectuelle plutôt restreinte. De cet organe militaire officiel à une revue de propagande politique, il n'y a peut-être qu'un pas. Celle-ci s'enorgueillit dans un avant-propos datant de 1949 d'avoir à sa tête, pour coordonner son édition, un normalien agrégé d'histoire et de géographie. Son ambition est de couvrir la totalité des problématiques rencontrées dans l'espace allemand par l'armée française, afin de 
mettre en relief, je cite: «La grandeur de l'œuvre que nous - c'est-à-dire le gouvernement militaire - avons accomplie en Allemagne ».

13 L'activité de Michel Eude, au sein de cette publication, relève essentiellement de la coordination éditoriale. Il n'y rédigera que deux articles dans la rubrique « Politiques et économiques » ainsi qu'une vingtaine de notices, dans la rubrique «Chronologies allemandes ", rendant compte à chaque numéro de l'évolution sur le plan intérieur et extérieur de l'Allemagne sous occupation alliée. On remarque ici un certain évitement des publications sous forme d'articles de synthèses ou d'articles problématiques et un choix délibéré - puisque ce dernier est directeur de la revue - de se limiter à des publications brèves sous forme de notice. Cet aspect formel du travail de Michel Eude est également présent dans ses publications dans les $A H R F$, distillées en quelque sorte au compte-goutte de son obsession rigoureuse du détail. Le plus souvent, ses articles relèvent davantage de l'exposé de détails et d'indications que d'un article de réflexion. C'est pourquoi je me suis permise de m'attarder quelque peu sur cette expérience à la fois personnelle, professionnelle et littéraire de Michel Eude qu'il me semble important de rattacher à son action au sein de la SER. Son envergure historique se trouvant limitée à l'intérieur même du corps textuel des informations qu'il rédige.

Cette composante militaire du parcours de Michel Eude est présente également dans l'histoire familiale de ce dernier. Fils d'officier, il épousera le rejeton d'une famille militaire. Il s'agit même d'une double alliance entre ces deux familles - comme il était assez fréquent - puisque sa sœur épousa le frère de son épouse. Son beau-père, le lieutenant général Goislard de Monsabert, fut l'auteur en 1950 d'une publication intitulée Il faut refaire l'armée française ${ }^{6}$ destinée à restaurer la " grandeur » de l'armée, en pleine crise de légitimité depuis la capitulation de juin 1940. Cet ouvrage à la gloire de l'armée française insiste en outre sur le rôle directeur que doit réoccuper la France sur la scène internationale. On retrouve ici dans la famille par alliance de Michel Eude, les préoccupations nationales et idéologiques qui animent la division de l'Information $\mathrm{du}$ gouvernement militaire de la zone française d'occupation. Force nous est de constater encore le fossé intellectuel qui sépare Michel Eude de l'élite montante de la SER dont Albert Soboul est une sorte de parangon, déployant l'histoire de la Révolution française à la lumière d'un engagement politique et civique fortement ancré à gauche. Par ailleurs, Michel Eude a rédigé avec son beau-frère Jacques de Monsabert un ouvrage «historico-familial », Les Goislard de Monsabert. Révolution et Restauration ?. Le titre, l'édition à compte d'auteur et la composition formelle assimilent cet ouvrage à un ensemble de publications familiales à vocation généalogique éloignant encore un peu plus Michel Eude des préoccupations de ses collègues révolutionnaires qui dressent alors de grandes synthèses de l'histoire politique et sociale de la Révolution française.

Ce contexte familial et cette trajectoire personnelle forment un arrière-plan hérité et choisi qui conditionne la production historique de Michel Eude, et expliquent notamment le caractère strictement institutionnel et politique de ses travaux. De plus en raison de l'importance de la composante militaire de sa biographie, il est significatif que ce dernier, à travers l'étude descriptive et minutieuse qu'il a dressé des rouages du Comité de Sûreté générale, se soit exclusivement intéressé aux services de police et de renseignements sous la Révolution. Il en est de même, pour ce motif récurrent des complots qui, selon ses recherches, rythment la chronologie révolutionnaire.

Il convient à présent de se pencher plus sérieusement sur les travaux de cet historien qui va trouver dans les AHRF le principal exutoire de sa production historique. 
Il est toutefois malaisé de dresser une vision d'ensemble du travail de Michel Eude car d'un point de vue formel, on dispose d'un corpus assez hétérogène : articles et notices publiés dans les AHRF mais aussi les Annales de la Manche et les Annales de Normandie de 1932 à 1984, la direction et édition du dernier tome de Paris sous la Terreur en 1964, une collaboration à l'ouvrage dirigé par Jacques Aubert en 1981, L'État et sa police en France (1789-1914); à la même époque il rédige plusieurs notices pour l'Encyclopedia Universaliset l'ouvrage familial des Goislard de Monsabert qui paraît en 1984. Sur le fond, il convient de remarquer l'absence d'une thèse que Michel Eude, pensionné par le CNRS, n'achèvera jamais, ainsi que l'absence d'un ouvrage individuel concernant les recherches auxquelles il a consacré sa vie, notamment son étude du Comité de Sûreté générale. Seules les AHRF offrent un débouché national, voire international, aux travaux de cet historien.

18 Michel Eude a pourtant préparé sa thèse à l'Institut d'histoire de la Révolution française, dans un contexte historiographique en pleine effervescence. En 1958, Marcel Reinhard, alors directeur de l'Institut fait paraître un article dans l'importante revue historique The Journal of Modern History afin de faire connaître les activités du centre. Il relate en premier lieu la thèse de Michel Eude qui se veut, je cite, une "étude exhaustive du Comité de Sûreté générale, institution plus célèbre que bien connue ", ensuite Marcel Reinhard en vient à traiter des travaux de deux membres éminents de la SER à savoir « les armées révolutionnaires » de Richard Cobb et la «thèse consacrée à une institution fameuse et obscure » d'Albert Soboul... sur les « sections parisiennes en l'an II ». Sur l'ensemble de ces thèses, Marcel Reinhard conclut : « Études d'institutions, mais études sociales simultanément et même nécessairement ${ }^{8}$. Il est vrai qu'on peut constater une continuité dans l'effort méthodologique de ces "grands travaux de l'IHRF » où tant Michel Eude qu'Albert Soboul ou Richard Cobb effectuent un véritable retour aux sources, dépouillant des corpus d'archives primaires conséquents. Cependant, Marcel Reinhard évoque le lien essentiel existant entre l'étude institutionnelle et la société que cette dernière donne à voir, or si celle-ci se matérialise à nos yeux dans les travaux de Soboul et Cobb, elle constitue justement la limite des travaux de Michel Eude. Car, à en juger par le fonds qu'il nous a légué, il s'est là encore arrêté à une étude strictement institutionnelle et administrative des fonctionnements du Comité de Sûreté générale. L'inachèvement de sa thèse réside en partie, je pense, dans cette absence de focale sociale envers une institution qui laisse pourtant deviner un lourd contentieux socio-politique. Néanmoins, les archives ont été scrupuleusement dépouillées, offrant un outil de travail et un corpus sériel précieux à quiconque voudrait travaillait sur le sujet, et plus précisément sur les institutions et le personnel de la «Terreur ». Michel Eude semble toutefois avoir buté sur la concrétisation de cet outil en un ouvrage de réflexion plus globale sur le Comité de Sûreté générale dans la société française au temps de la Convention montagnarde. En revanche, on peut sentir l'historien beaucoup plus à l'aise dans la direction du dernier volume de Paris pendant la Terreur paru en 1964, grande compilation érudite des rapports des agents secrets du Ministère de l'Intérieur. Michel Eude a pu exprimer cette passion du détail que l'on retrouve dans l'ensemble de son œuvre et mettre sa connaissance archivistique et prosopographique de l'architecture administrative montagnarde au service de ce recueil de sources, qui, dans le prolongement de la direction de Pierre Caron, selon Michel Eude dans son avant-propos, "s'est efforcé, dans les notes infra-paginales, d'identifier les personnages et d'élucider les faits dont parlent les observateurs du ministère $"{ }^{9}$. En parcourant ses publications dans les AHRF, on retrouve ce goût en soi 
de la minutie historique et du détail factuel. Il publiera également en 1918 un volume de tables "permettant aux historiens, selon lui, d'utiliser plus commodément les six volumes de Paris pendant la Terreur $"{ }^{10}$. Dans ce cadre formel et utilitaire, Michel Eude a pu exprimer les qualités d'un historien qui se montre, selon Suratteau, «aussi discret que savant ${ }^{11}$.

Cette composante minutieuse se retrouve également dans sa contribution au colloque L'État et sa police en France (1789-1914) paru en 1919. Cette contribution porte bien évidemment sur le Comité de Sûreté générale et traite essentiellement le sujet au travers des rouages de l'institution et des personnes l'ayant animée. Cependant, cette qualité de rigueur factuelle constitue également la limite de son analyse historique à en croire la critique émanant des recensions de l'ouvrage. Critique que l'on retrouve notamment en 1982 sous la plume de l'historien écossais Robert Anderson dans The Journal of Modern History, pour qui, Michel Eude se borne à décrire l'organe policier du Comité de Sûreté générale, faisant de sa communication une compilation de détails plus qu'une réelle réflexion de fond sur le problème posé par cette institution ${ }^{12}$. Anderson attaque d'ailleurs la cohérence même du colloque dont le titre " prometteur » selon ses mots a donné lieu à un ensemble de communications de qualité aléatoire et plutôt superficielle. La plupart des recensions du colloque sont d'ailleurs assez réservées et ne mentionnent que poliment la communication de Michel Eude ${ }^{13}$. Une entreprise qui ne fut donc pas vraiment couronnée de succès... et pourtant pour ce dernier, c'était le lieu possible de l'expression d'un sujet de recherche de toute une vie.

Revenons donc à notre vision d'ensemble sur les publications de Michel Eude. Il s'agit d'un corpus disparate dont la réception laisse quelque peu à désirer. Trois moments forts rythment pourtant les publications de cet historien dans les AHRF, tels les trois âges de sa recherche.

21 Celles-ci débutent dans les années 1930, avec entre autre un article important sur la Commune robespierriste paru entre 1932 et 1936. On l'a vu, il s'agit de la première entrée de Michel Eude dans l'univers intellectuel des AHRF et l'univers social et intellectuel de la SER. C'est dans cet article publié également par la Bibliothèque d'histoire révolutionnaire de la SER, que sa recherche menée à la fondation Thiers sur la Commune robespierriste trouve donc son aboutissement. Il ne fit paraître ensuite que quelques notices dans la rubrique «Glanes » entre 1935 et 1937 et interrompt son activité éditoriale le temps de la Seconde Guerre mondiale.

Le deuxième "grand moment " éditorial participe de la dynamique générale de la revue au cours des décennies 1950-1970. Michel Eude renoue alors avec la publication d'articles de fond consacrés essentiellement au personnage de Robespierre et aux réseaux qui l'entourent. Il publie notamment «La politique de Robespierre en 1792 d'après "Le Défenseur de la Constitution" » en janvier-mars et avril-juin 1956. Cet article, long de plus d'une cinquantaine de pages, traite de la stratégie politique que permet de soutenir Le défenseur de la Constitution, organe de presse animé par Robespierre dans le courant de l'année 1792. Michel Eude entend ici rebondir sur la publication des CEuvres complètes de Robespierre par la SER. Une fois de plus, l'activité de la SER offre à ce chercheur une dynamique d'expression et de débat dont les AHRF se font la tribune. Les décennies 1950-1960 marquent la période la plus féconde de Michel Eude, alors motivé par la rédaction de sa thèse en cours et sa mutation à la Faculté de lettres de Rouen comme maître-assistant, sur recommandation de Marcel Reinhard et de Jean Vidalenc. De 1964 à 1969, Michel Eude élargit quelque peu ce spectre de 
préoccupation «robespierriste» et explore, par le biais de publications dans les rubriques "Articles » et "Mélanges ", différents aspects de la mécanique policière et judiciaire de la Terreur, revenant notamment sur le Comité de surveillance de l'Assemblée législative, prélude du Comité de Sûreté générale, ou encore l'affaire Catherine Théot, dans lesquels il évoque toujours en arrière-plan une France sous étroite surveillance.

Le troisième "temps fort " a lieu entre 1981 et 1985, date de sa dernière grande publication : un article de synthèse sur le Comité de Sûreté générale ${ }^{14}$, pierre angulaire de son activité de chercheur, "sujet qui l'avait passionné pendant un demi-siècle " ${ }^{15}$ comme le rappelle Jean-René Suratteau. On ne peut s'empêcher de voir dans cet article une sorte de bilan de toute une vie de recherche, sorte de chant du cygne de l'activité historique de Michel Eude qui s'éteindra quelques années plus tard. C'est alors, au terme de son parcours d'historien, que pour la première fois, il ne se contente pas d'un exposé minutieux de détails factuels, mais se lance à énoncer clairement sa vision de la Terreur, celle d'un « régime de tension et de compression », "régime que [selon lui] l'organisme humain est incapable de supporter $"{ }^{16}$.

24 Pendant ces trois épisodes les publications dans les AHRF ont permis de donner une visibilité aux recherches de Michel Eude, mais ont également pallié les lacunes bibliographiques de ce dernier, allant jusqu'à se faire le support d'un condensé de la thèse inachevée.

Un dernier aspect de sa trajectoire peut être également évoqué par le biais de la sociabilité intellectuelle de la SER. Michel Eude, à partir de son retour en France en 1952, assiste régulièrement aux réunions de la Société, participant volontiers aux débats, présentant également une communication à l'assemblée générale du 15 mars 1964 sur « Le comité de surveillance de l'Assemblée législative, 1791-1792 ", à l'issue de laquelle vont notamment participer au débat Soboul et Reinhard ${ }^{17}$. Ici encore les décennies 1950-1960 marquent le temps fort de la participation de Michel Eude aux assemblées générales de la SER. On est alors dans un contexte d'effervescence intellectuelle au sein des études robespierristes et plus généralement au sein des études révolutionnaires. Dans son ouvrage Combats pour la Révolution, Michel Vovelle parle d'un véritable épanouissement de l'école jacobine pour cette période où triomphe avec les travaux d'Albert Soboul une lecture sociale de la Révolution française dans le prolongement des études impulsées par Mathiez et Lefebvre ${ }^{18}$. Michel Eude est continûment mentionné dans les procès-verbaux des assemblées générales comme intervenant dans les débats qui suivent les nombreuses communications présentées lors de ces réunions. Cependant, dans le cas de communications historiographiques et plus précisément de celles portant sur l'héritage du marxisme ou du socialisme dans les études révolutionnaires, à l'instar des communications pluridisciplinaires portant notamment sur l'usage d'outils empruntés aux autres sciences humaines, Michel Eude pourtant présent, n'intervient pas dans les débats. C'est le cas notamment lors de la communication d'Albert Soboul sur «Robespierre ou les contradictions du jacobinisme ", présentée en 1977, mais l'on ne peut saisir si c'est l'exposé ou l'exposant qui suscite ici cette attitude ${ }^{19}$. Le silence de Michel Eude sur ces types de sujets plus polémiques ou avant-gardistes peut n'être qu'une pure coïncidence, mais mérite toutefois d'être remarqué par contraste avec sa participation continue à l'ensemble des autres communications auxquelles il assiste. Dès la fin des années 1970, Michel Eude 
s'éclipse des assemblées générales de la Société très certainement pour des raisons de santé.

Dernière remarque sur la participation de Michel Eude à la vie de la SER : il entre au comité directeur en 1940 pour une brève année avant la cessation d'activité de la société tourmentée par le régime de Vichy, mais il ne sera pas reconduit en 1946 lorsque celle-ci se reforme une fois la guerre finie. Ainsi, durant ces vingt années de présence et de participation aux réunions de la SER, Michel Eude n'assurera pas non plus de mandats au sein de la SER, passage obligé des grandes figures de la société, il ne fait donc pas partie des membres actifs de la SER, ce qui peut d'ailleurs être mis en rapport avec la dominante marxiste des mandataires de la société comme Ernest Labrousse ou Albert Soboul. Cette participation réservée contraste une fois de plus avec ces grandes figures tutélaires de la SER, mais son personnage méconnu a le mérite d'offrir une posture alternative des historiens dans le monde des études robespierristes, la possibilité justement d'une posture décalée, un peu terne et laborieuse, à l'écart des projecteurs.

Enfin, pour conclure sur cette société aujourd'hui centenaire, l'étude de ce type de personnage permet de ne pas cristalliser notre attention sur un acteur, mais de s'intéresser également à son milieu. Dans cette étude de cas, on peut observer la SER et les AHRF jouer le rôle qu'elles tiennent encore de nos jours, véritable plates-formes intellectuelles qui intègrent des chercheurs d'envergure et de postures différentes, assurant à chacun un débouché adapté à l'offre historique qu'ils proposent et élaborent. Sans y avoir joué un rôle essentiel, Michel Eude nous permet donc de pointer ici la scène significative constituée par la SER dans la «fabrique de l'histoire » de la Révolution française.

\section{Liste des publications de Michel Eude dans les Annales historiques de la Révolution française}

\begin{tabular}{|c|c|c|c|}
\hline Titre & Rubrique & Pagination & Année \\
\hline La Commune robespierriste & Article & $\begin{array}{l}(1931) \text { p. } 557, \\
(1933) \text { p. } 411-415 ;(1934) \text { p. } \\
513-547 \text { et } 518-556 ;(1935) \text { p. } \\
131-161 ; \\
(1936) \text { p. } 139-316\end{array}$ & 1931-1936 \\
\hline Buissart et Robespierre & Glane & p. 460 & 1935 \\
\hline
\end{tabular}




\begin{tabular}{|c|c|c|c|}
\hline La diffusion du manifeste de Brunswick & Glane & p. 469 & 1935 \\
\hline Sur une allusion du Vieux Cordelier & Glane & p. $460-461$ & 1937 \\
\hline \multicolumn{4}{|l|}{ Parenthèse de la seconde guerre mondiale } \\
\hline Robespierre et le miniaturiste Thoüesny & article & p. 193-101 & 1955 \\
\hline $\begin{array}{l}\text { La politique de Robespierre en } 1792 \\
\text { d'après Le Défenseur de la Constitution }\end{array}$ & article & p. $1-18$ & 1956 \\
\hline Breteuil, Bombelles, Castiers en 1791 & article & p. 36-53 & 1961 \\
\hline $\begin{array}{l}\text { Le Comité de surveillance de l'Assemblée } \\
\text { législative, 1791-1792 }\end{array}$ & article & p. $119-148$ & 1964 \\
\hline $\begin{array}{l}\text { Robespierre a-t-il voulu faire destituer } \\
\text { Fouquier-Tinville? }\end{array}$ & Mélange & p. $66-71$ & 1965 \\
\hline Maure ou Pottofeux? & Mélange & p. $413-419$ & 1966 \\
\hline $\begin{array}{l}\text { Points de vue sur l'affaire Catherine } \\
\text { Théot }\end{array}$ & article & p. $606-619$ & 1969 \\
\hline \multicolumn{4}{|l|}{ Parenthèse des années 1970} \\
\hline $\begin{array}{l}\text { Une interprétation « non- } \\
\text { mathiézienne » de l'affaire de la } \\
\text { Compagnie des Indes }\end{array}$ & article & p. $139-161$ & 1981 \\
\hline La loi de Prairial & article & p. 554-559 & 1983 \\
\hline $\begin{array}{l}\text { Le Comité de Sûreté générale en } \\
1793-1794\end{array}$ & article & p. $195-306$ & 1985 \\
\hline
\end{tabular}

\section{NOTES}

1. Le Fonds Michel Eude est constitué d'une vingtaine de cartons consacrés à l'institution du Comité de Sûreté générale, il s'agit de la copie manuscrite, intégrale ou partielle, des fonds relatifs au Comité et conservés au Centre historique des Archives nationales (sous-série F7). Cette somme rassemblée par Michel Eude a été soumise à plusieurs classements thématiques, elle comporte trois grandes subdivisions :-Les "Affaires particulières " comprenant l'ensemble du fichier des suspects du Comité de Sûreté générale (sous série F7).-Les «Arrêtés du Comité de sûreté générale » comprenant les mandats d'arrêt, mandats d'amener, mandats de perquisitions et la correspondance du Comité (sous série F7, AFI*).-Le "Mélange » comprenant des archives diverses relatives aux députés, à la commission des XXI, aux passeports (sous série F7, BB).

2. Jean-René Suratteau, Hommage à Michel Eude (1909-1988), AHRF, 1989, p. 75.

3. Michel Eude, La Commune robespierriste, diplôme d'études supérieures d'histoire moderne à la faculté des Lettres de Paris, 1932.

4. On trouvera une liste détaillée de ses publications dans les AHRF à la fin du présent 
article.

5. Réalités allemandes, Haut Commissariat de la République française en Allemagne, Direction générale des affaires politiques, Information, Bad Godesberg, 1949-1952. Cette revue sera publiée par la suite sous le nom Allemagne d'aujourd'hui, Réalités allemandes, Paris, PUF, 1953-1957.

6. Général de Goislard de Monsabert, Il faut refaire l'armée française, SES, 1950, $180 \mathrm{p}$.

7. Michel Eude et Jacques Monsabert, Les Goislard de Monsabert. Révolution et Restauration, Hastingues, J. de Goislard de Monsabert, 1984, 139 p.

8. Marcel Reinhard, "L'Institut d'histoire de la Révolution française à la Sorbonne ", The Journal of Modern History, vol. 30, $\mathrm{n}^{\circ}$ 4, décembre 1958, p. 355.

9. Michel Eude, «Avant-propos ", Michel Eude (dir.), Paris pendant la Terreur, tome VI, Paris, Klincksieck, 1964, p. II.

10. Id., p. III.

11. Jean-René Suratteau, dans Michel Eude (dir.), Paris..., op. cit., p. 75.

12. Robert D.Anderson, «L'État et sa police en France (1789-1914) by Jacques Aubert », The Journal of Modern History, vol. 54, n 4, décembre 1982, p. 794.

13. J. P. T. Bury, «L'État et sa police en France (1789-1914) », The English Historical Review, vol. 96, $\mathrm{n}^{\circ} 380$ (Jul. 1981), p. 659-660.

14. Michel Eude, «Le Comité de Sûreté générale en 1793-1794 », AHRF, 1985, p. 295-306.

15. Jean-René Suratteau, Ibid, p. 77.

16. Michel Eude, «Le Comité... », art. cit., p. 396.

17. Le procès-verbal de la séance mentionne que sa communication fut «chaleureusement applaudie ", mais ne le sont-elles pas toutes?

18. Michel Vovelle, Combats pour la Révolution, Paris, La Découverte, 1993, p. 63.

19. Il semble que ces deux personnages entretenaient une relation plutôt hostile.

\section{RÉSUMÉS}

À l'ombre des grandes figures qui ont fait la Société des études robespierristes, il est possible de saisir les bribes de trajectoires individuelles moins audacieuses, mais tout aussi signifiantes des évolutions de cette société savante. Ainsi, l'historien du Comité de Sûreté générale, Michel Eude (1909-1988), sociétaire pendant près d'un demi-siècle et conservateur en retrait de l'école marxiste, offre un biais alternatif pour étudier la SER: son relatif anonymat et sa posture intellectuelle permettent de saisir celle-ci comme un milieu contrasté, opérant la synthèse entre différentes personnalités scientifiques et politiques. Son cheminement d'historien entre en constante relation avec le monde des études robespierristes qui fut tout à la fois pour celui-ci un vecteur d'initiation universitaire, une plateforme éditoriale et enfin et surtout le support essentiel d'une sociabilité savante.

In the shadow of the major figures who created the Société des études robespierristes, it is possible to catch glimpses of less spectacular individual trajectories, though these are no less significant for the evolution of this learned organization. Thus the historian of the Comite of General Security, Michel Eude (1909-1988), a member of the organization for nearly a half century and a retired conservator of the Marxist school, offers an alternative way of studying the SER: his relative anonymity and his intellectual status demonstrates this contrasting milieu, effecting a synthesis between different scientific and political personalities. His evolution as an 
historian brought him into constant contact with the world of the "études robespierristes " which was at the same time a vector of university initiation, an editorial platform, and finally, and above all, the essential support of a scholarly sociability.

INDEX

Mots-clés : Michel Eude, Société des études robespierristes, Institut d'histoire de la Révolution française, Comité de Sûreté générale, Robespierre, historiographie

\section{AUTEUR}

\section{CLYDE PLUMAUZILLE}

IHRF - Université Paris I 35, rue Linné 75005 Paris, clyde-marlo.plumauzille@orange.fr 